\title{
The Evaluation of Operating Posture in Typing the QWERTY Keyboard on PDA
}

\author{
Han-Chi Hsiao, Fong-Gong Wu, Ronald Hsi, Chih-I Ho, Wen-Zhou Shi, \\ and Chien-Hsu Chen \\ Department of Industrial Design, National Cheng Kung University, 1, \\ Ta-Hsueh Road, Tainan, Taiwan 70101, Taiwan \\ hedyhanchi@yahoo.com.tw
}

\begin{abstract}
In this research, we observed the user's posture while using PDA. 30 participants typed the keys with standard QWERTY keyboard on the PDA. At the end of the experiments the participants who have professional background in design were asked to complete an open-ended questionnaire, which is in order to evaluate the usability of the PDA. In the final, we presented the suggestion of design criterion for keyboard, as to provide the references for future PDA design. The statistical result of the posture while using the PDA revealed that the most users held PDA with both hands and pressed keys with both thumbs. The findings in this research suggest that when we design small input devices such as PDA in the future, the stability of the keyboard usage should be taken into considerations to enhance its input performance and improve user experience.
\end{abstract}

Keywords: Letters Key Design, PDA Typing Posture.

\section{Introduction}

The use of digital mobile devices is becoming popular with the fast growing economy, many business workers have grown used to working with a PDA to do computer related work. The most common work done on a PDA is text input, which includes the input of electronic mails or SMS text messages. Many past researches had focused on the muscle and bone injury caused during text input using a keyboard: Serina and others [1] found that incorrect wrist and forearm posture during text input is a risk to indisposed upper limb muscle and bone; Straker and others[2]did research on the comparison of text input posture between the use of desk top computers and laptop computers back in 1997, along with the effect on typing posture change under different types of working environment; Kotani and others[3] brought forward the effect different horizontal positions of keyboards have on forearm and wrist posture during typing; the further the keyboard is from the user the smaller the ulnar deviation angle and the larger the back bending angle, a supporting mattress could help with the back bending problem. As for posture analyzing, Baker and others [4] brought forward the study of kinematics of fingers and hands during computer operation.

Colle et al.[5]studied the features of the keys, focusing on the experiments on the different sets of key sizes $(10,15,20,25 \mathrm{~mm})$ and gaps $(1.3 \mathrm{~mm})$ between virtual number 
keys. The best size was found to be between $15 \sim 20 \mathrm{~mm}$, and no obvious effect was found in the gap differences. Gong and Tarasewich[6]designed a new alphabetical distributed input pattern to be used on mobile phone keypads. Sears et al. [7]studied the effects of different key sizes and user operation on virtual key data input on mobile devices. Many others studied different types of keys [8][9][10][11], including varies virtual and concrete keypads and small-sized concrete keypads designed for mobile devices. Li et al.[12]studied single-handed input keypad design. Kim et al.[13]studied the use of small QWERTY keypads on portable electronic devices. Wobbrock et al.[14]studied the integration of joystick control and touch screen as an input pattern on small devices. As for key operation efficiency, Silfverberg el al.[15] established a formula predicting text input speed on mobile phones. Mizobuchi et al.[16]focused on the connection between walking speed and the level of mobile phone text input difficulty.

From earlier researches, many human-engineering related problems on standard computer keyboard input operation have already been discovered, especially problems with diseases caused by long-term incorrect posture. However, these standard keypad arrangements are still very common in the market today. With increased functions and decreased sizes on mobile devices, small-sized standard keypads are being used, especially on personal digital assisting devices. Using a large number of keys in a limited space with a repetition of incorrect posture often causes injury around hand areas (fingers, wrists, forearms etc.). For fingers, past studies focused on thumb posture during mobile phone operation [17], thumb adduction/abduction and palm flexion/extension problems were found. Since no literature has fully covered the study of PDA keypad operation posture, our research will focus on the observation of vertical QWERTY keypad operation on text input, with further advice on key designs.

\section{Method}

The NOKIA E71 model has been chosen for our experimental observations. There are two major stages to the research. The first stage covers the observation of PDA operation posture of users and the later stage focuses on the connection between PDA operation posture and the pain caused. Our goal is to conclude suitable operation posture for PDA and recommend better PDA key designs.

\subsection{Experiment A: PDA Operation Posture Observation}

Experiment A observes the different types of PDA operation posture. That is, observing vertical PDA users typing English on QWERTY keypads, focusing on the operation posture while standing and sitting. The independent variable being the operation style (standing, sitting), the dependent variable being hand posture types, the controlled variable being gender, distance between screen and participant, and experimental environment. Data obtained from the experiment is handed to four human-engineering specialists for further classification according to posture.

\subsubsection{Subjects}

Thirty subjects were obtained from National Cheng-Kung University. They were asked to be right-handed with no RSI or other hand injuries, to frequently input text, 
and to have normal eye-sight with or without lenses. All subjects need to sign an agreement prior to the experiment. The 30 subjects participating in the experiment all came from a design background, between the ages of 21-37 with an average age of $24.7 \pm 3.2$, and an average palm width of $78.42 \mathrm{~mm}(\mathrm{SD}: 6.55)$. Subjects include 15 male, with an average age of 26 (SD: 1.08) and average palm width of $84.07 \mathrm{~mm}$ (SD: 0.80 ); 15 females with an average age of 24 (SD: 0.47) and an average palm width of $72.77 \mathrm{~mm}$ (SD: 0.85).

\subsubsection{Experimental Procedure and Measurements}

Describe the goal and task of the experiment to subjects in advance and ask for basic information and measure palm width. Then ask subjects to operate on the PDA models in the two operation styles of standing and sitting. The subjects are asked to input English text once for the two operation styles, with the text given to them on a Microsoft Power Point 2003 file $460 \mathrm{~cm}$ in front of them. The operation style order and one of the two English text is given in random. 15 subjects are asked to stand first and 15 to sit. The distance between standing and sitting subjects is $91.7 \mathrm{~cm}$.

Prior to the experiment, researchers describe experiment procedures to the subjects and do test runs. Photographs are taken to keep a record of posture before and after operation. Subjects are asked to input an English text with a model of the NOKIA E71 mobile phone and are asked to read each letter as they type to record the input speed. For example, when inputting the sentence "Do not laugh", subjects will need to read "D" after typing it, read "o" after typing "o" and say "space" after entering a space and so on. During the standing operation experiment, subjects are asked to operate in a natural and comfortable manner. During the sitting operation experiment, a table of $73 \mathrm{~cm}$ in height, $69 \mathrm{~cm}$ in width and $59.5 \mathrm{~cm}$ in depth is placed in front of the subjects. The subjects are asked to operate in a natural and comfortable manner with or without hands on the table. During the experiment, researchers record the sitting experiment with a CANON IXU 600 digital camera and the standing experiment with a CANON IXU 800 digital camera, focusing on hand movement operation posture. Another Panasonic digital camera is used to record hand posture before and after operation of both styles; shooting a hand-held posture photograph from each of the front angle, upper angle, right angle, and left angle. Lastly, the subjects are asked to fill out a questionnaire concerning PDA keypad operation analysis and related questions.

\subsection{Experiment B: Observation of the Connection between Various Posture and Pain}

Experiment $\mathrm{B}$ focuses on the connection between various posture and pain. According to the hand-held and thumb typing posture concluded from experiment A, study the connection between different PDA typing posture and pain(including index finger support, little finger support, ring finger support, and four finger support). The independent variables include index finger support posture, little finger support posture, ring finger support posture, and four finger support posture; the dependent variables include the level of pain in the thumb, wrist, elbow, forearm, shoulder and neck; the control variables include the distance between drawing board and subjects, and experimental environment. Lastly, data is analyzed using repeated measures. 


\subsubsection{Subjects}

Thirty subjects participated in the experiment, with 14 male and 16 female. They are all right-handed with no RSI or other hand injuries. They are familiar with text input and all have normal eyesight. The average height for male subjects is $174 \pm 6.98 \mathrm{~cm}$, average left hand length being $186.21 \pm 17.78 \mathrm{~mm}$ and right hand length being $183.25 \pm 17.41 \mathrm{~mm}$; average left palm width being $80.32 \pm 5.01 \mathrm{~mm}$, right palm width being $82.21 \pm 4.61 \mathrm{~mm}$. The average height for female subjects is $162.5 \pm 5.39 \mathrm{~cm}$, average left hand length being $172.19 \pm 8.83 \mathrm{~mm}$ and right hand length being $171.53 \pm 9.70 \mathrm{~mm}$; average left palm width being $73.5 \pm 3.55 \mathrm{~mm}$, right palm width being $74.86 \pm 3.61 \mathrm{~mm}$.

\subsubsection{Experimental Procedure and Measurements}

First explain the goal and tasks of the experiment to the subjects and ask them to fill out basic information and sign an experiment agreement. Then, subjects must support the PDA in certain posture to input an English text, one specific posture for each text message. Specific supporting posture includes index finger support, little finger support, ring finger support, and four finger support. Subjects draw out the posture order before typing, each subject is required to type four different texts with a different posture for each. The texts are four short descriptive articles chosen from the website: http://health.discovery.com, each contain about 179-184 words without punctuations. The procedure for each posture has a time limit of six minutes. Since PDA is a mobile device that is mostly used standing, hence subjects are asked to stand for the experiment. During the input process, subjects are allowed to move their bodies but not their hand posture. Researchers will record the standing posture by taking a photograph every 2 minutes with a digital camera on the front-right and the front side of the subject. When one text is done, researchers make a record of the number of words, and the subject is asked to complete a questionnaire about the pain caused by the posture. Analysis is done on five parts of the body (thumb, wrist, elbow, forearm, shoulder and neck), rating from $0 \sim 10$, where 0 stands for no pain and 10 stands for the most pain. After completing the analysis for each of the four posture, researchers will measure the palm width and hand length of the subject.

\section{Results and Discussion}

\subsection{Experiment A: Observation Results of PDA Operation Posture Types}

In this subject we have differentiated PDA operation posture into standing and sitting, hand-held posture into left hand support, right hand support and two hands support. With a standing posture, $90 \%$ (27 subjects) supported the PDA with two hands, $6.7 \%$ (2 people) supported with left hand, and $3.3 \%$ (1 person) with right hand; with a sitting posture, $83.3 \%$ (25 people) supported with two hands, $6.7 \%$ (2 people) supported with left hand, $3.3 \%$ (1 person) with right hand, and $6.7 \%$ (2 people) set it on the desk for operation.

There are four posture for pressing the keypad (Fig. 1): right thumb pressing, right index finger pressing, two hands thumb pressing and two hands finger pressing. With a standing posture, $13.3 \%$ (4 people) pressed the keypad with right thumb, 3.3\% (1 person) pressed with right index finger, $73.5 \%$ (25 people) pressed with two 
Table 1. Handheld posture

\begin{tabular}{lrr}
\hline & Standing & Sitting \\
\hline Two hands support & 27 & 25 \\
Left hand support & 2 & 2 \\
Right hand support & 1 & 1 \\
Lying on desk & 0 & 2 \\
Total & 30 & 30 \\
\hline
\end{tabular}

Table 2. The Pressing and support posture

\begin{tabular}{lrr}
\hline Pressing posture on keypads. & Standing & Sitting \\
\hline Right hand thumb pressing & 4 & 3 \\
Right hand index finger pressing & 1 & 2 \\
Two hands thumb pressing & 25 & 24 \\
Two hands all fingers pressing & 0 & 1 \\
Total & 30 & 30 \\
\hline Upper edge support posture. & Standing & Sitting \\
\hline Two hands index fingers & 8 & 6 \\
Single hand index finger supporting upper edge of PDA & 5 & 3 \\
No upper edge suppor & 17 & 21 \\
Total & 30 & 30 \\
\hline Lower edge support posture. & Standing & Sitting \\
\hline Middle finger support lower edge & 3 & 2 \\
Ring finger & 9 & 12 \\
No lower edge support & 6 & 9 \\
total & 12 & 7 \\
Total & 30 & 30 \\
\hline
\end{tabular}

thumbs in turn; with a sitting posture, $8.8 \%$ (3 people) pressed the keypad with right thumb, 5.9\% (2 people) pressed with right index finger, $70.6 \%$ ( 24 people) pressed with two thumbs in turn, and $2.9 \%$ (1 person) pressed with two index fingers in turn.

During PDA operation, users often use finger support to gain stability, we will look at upper edge support and lower edge support: With a standing posture, $16.7 \%$ (5 people) uses an index finger to support the upper edge of the PDA, 26.7\% (8 people) use two index finger, $56.7 \%$ (17 people) did not support the upper edge; With a sitting position, $10 \%$ (3 people) used a middle finger to support the lower edge, $30 \%$ (9 people) used a ring finger, $60 \%$ ( 9 people) used a little finger, and $13.3 \%$ ( 7 people) did not support the lower edge.

To sum up, we have concluded 4 types of holding posture from the observation in experiment A (Fig. 2). They are two hand holding posture, thumb pressing, upper edge support, and lower edge support. The results show that the two hand holding 
posture and two thumb typing in the main stream for PDA operation. During the sitting and standing posture, subjects used two index fingers to support the upper edge of PDA or simply no upper edge support; With a standing posture, subjects either support the lower edge of PDA by using ring fingers or simply did not support the lower edge; with a sitting posture, many support the lower edge with ring finger and little finger to gain stability.

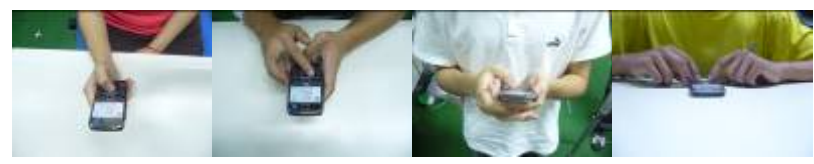

Fig. 1. Key pressing posture

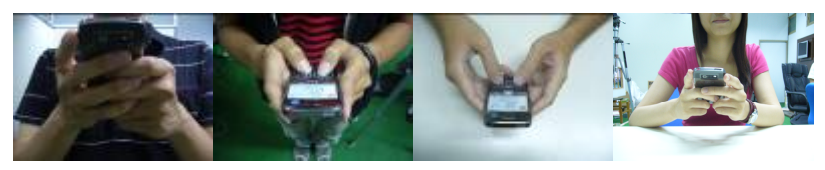

Fig. 2. Grip and stability posture

We looked at the connection between the various posture using $\chi^{2}$, and found a positive connection between handheld posture and fingers used for typing with both standing and sitting posture, a connection between upper edge support and lower edge support, a connection between handheld posture and whether forearm is leaning against table in a sitting posture, a connection between elbow support and hand support in a sitting posture, and a connection between elbow support and forearm learning against desk in a sitting position.

As for the keys, subjects generally commented on PDA keys being too small, causing a high error rate, and the large number of characters causes confusion in recognition. The clarity and choice of icons and the convenience of symbol and input language switching both need to be worked on, feedback sounds should be considered too; distance between keys needs to be clear for recognition and the keypad curving angle needs to avoid reflection. As for key arrangements, subjects declared that although the arrangement is similar to a computer keyboard, keys may be hard to find for non-computer users, hence training may be needed. If chord corresponding keys are used then comfort needs to be considered, an alphabetical order arrangement may be more appropriate than a computer keyboard arrangement. After learning and training, chord corresponding keys should be able to reach the same average speed as the standard keyboard arrangement, the key size problem could also be solved. As for the PDA width size, subjects suggested a width that is comfortable to be held singlehanded, so it is comfortable to operate with one or two hands. This could be shown on the LCD screen by a horizontal displacement mechanism. 


\subsection{Experiment B: Observation Results of the Connection between Posture and Pain During PDA Operation}

From experiment A, we found that most people take the posture of two hand holding and two thumb pressing, and users take different posture to support PDA to gain stability. Since mobile devices require a fast-speed data transfer, stability in hands is a must. According to the stability supporting posture in experiment A, Experiment B focuses on the connection between supporting posture and pain during speed input operation.

When supporting the lower edge of PDA with the index finger, the pain level caused from severe to mild goes from shoulder and neck(51.57), thumb(49.07), wrist(39.70), forearm(39.37), to elbow(37.47). When supporting the lower edge of PDA with the little finger, the pain level caused goes from shoulder and neck(47.23), thumb(42.73), forearm(37.37), wrist(35.83), to elbow(33.80). When supporting the back of PDA with four fingers, the pain level caused goes from shoulder and neck(53.23), thumb(40.70), forearm(40.43), elbow(33.63), to wrist(29.80). When supporting the lower edge of PDA with the ring finger, the pain level caused goes from shoulder and neck(53.30), forearm(40.73), thumb(39.53), wrist(38.60), to elbow(34.67).

We can see that with either of the four posture, should and neck pain level were the most severe, followed by forearm, wrist, and elbow. After observing the full-body photographs, we found that subjects often make changes with standing posture, the shoulder and neck pain is caused by the long-term standing posture and the head movement due to the need of viewing the screen and external information. However, whether the connection exists between viewing the screen to input data and the shoulder and neck pain, is yet to be verified. The thumb pain caused by speed input with a fixed posture is rated second in the pain level. From this we can see that most people input with their thumbs, this creates speed, but causes excess burden on the thumbs even only for a short period of input time. Forearm and wrist pain were rated third. This could be caused by the wrist and forearm turning inwards, causing discomfort during speed data input. Elbow caused the least pain, and this could be due to the support given by chest and waist when placing elbows on the two sides of the body.

Table 3. The consistency of pain analysis in the five areas. (LSD comparison afterwards)

\begin{tabular}{|c|c|c|c|c|c|}
\hline Area & Thumb & Wrist & Elbow & Forearm & Shoulder and Neck \\
\hline Thumb & & (C) $\triangle$ & (a) & & \\
\hline Wrist & (0) $\triangle$ & & & $\triangle$ & (C) $\triangle \bullet$ \\
\hline Elbow & (C) & & & & (C) $\triangle \bullet$ \\
\hline Forearm & & $\triangle$ & & & (C) $\triangle \bullet$ \\
\hline Shoulder and Neck & & (a) $\triangle \bullet$ & (a) $\triangle \bullet$ & (C) $\triangle \bullet$ & \\
\hline
\end{tabular}

(): when supporting PDA with index finger support, $\mathrm{p}<0.05$

$\triangle$ : when supporting PDA with four finger support, $\mathrm{p}<0.05$

•: when supporting PDA with ring finger support, $\mathrm{p}<0.05$ 
The repeated measures result shows no significant difference in the connection between thumb pain, wrist pain, elbow pain, forearm pain, shoulder and neck pain and the four supporting posture. Hence, the four posture do not cause significant pain in the five body areas. However, when testing the consistency of pain analysis in the five areas (Table 3), it shows a higher level of shoulder and neck and thumb pain in all except for the little finger support posture. Including when supporting PDA with index finger, thumb pain was more severe than the wrist and elbow pain, and shoulder and neck pain was more severe than the elbow, forearm, and wrist; when supporting PDA with four fingers, the thumb pain was more severe than wrist pain, shoulder and neck pain was more severe than elbow, forearm, and wrist pain, and the forearm pain more severe than wrist pain; when supporting with the ring finger, shoulder and neck pain was more severe than wrist, elbow, and arm pain. The reason why it shows no connection between supporting posture and pain, could be due to the length of operation, or the disturbance on analysis standards caused by analyzing five body areas for each posture. This means that subjects could forget the standard used for analyzing one posture from another. However, regardless of the supporting posture, thumb and shoulder and neck pain is easily seen during short-term fixed input posture compared to other body parts.

\section{Conclusion}

To make PDA portable, they are made small in size and light in weight. Due to its small-sized keys and limited space between the keys, users tend to use a single finger such as thumbs to press the keys. The size of PDA and its keys has a profound effect on the way people use them. We recommend designers to thoroughly consider the connection between key designs and operation posture.

It has become a trend for PDA to be small in size. Hence the only change we could make to avoid physical burden during operation is to improve key designs and input styles. We found that most users hold PDA with both hands and type with both thumbs. Supporting the PDA with the index finger, little finger, ring finger, and four finger postures do not cause obvious pain in thumb, wrist, elbow, forearm, and shoulder and neck. There is a higher level of shoulder and neck and thumb pain compared to the other parts with all supporting posture except for the little finger support. There is no connection between the pain level caused and supporting posture, it has more to do with operation time period. Disregard the supporting posture used, when operating for a short time period with fixed posture, thumb and shoulder and neck feels a higher level of pain than the other body areas. Hence, designs should try to avoid the excess use of thumbs. Designers could improve on key arrangement designs to change the operation style of PDA input, in order to avoid users pressing the keys with only their thumbs; also improve on the shape of PDA to increase stability, in order to avoid the pain caused due to excess pressing motion.

\section{Acknowledgments}

The authors would like to thank the National Science Council of the Republic of China for financially supporting this research under Contract No. NSC 97-2221-E006 -169 -MY3. 


\section{References}

1. Serina, E., Tal, R., Rempel, D.: Wrist and forearm postures and motions during typing. Ergonomics 42, 938-951 (1999)

2. Straker, L., Jones, K.J., Miller, J.: A comparison of the postures assumed when using laptop computers and desktop computers. Applied Ergonomics 28, 263-268 (1997)

3. Kotani, K., Barrero, L.H., Lee, D.L., Dennerlein, J.T.: Effect of horizontal position of the computer keyboard on upper extremity posture and muscular load during computer work. Ergonomics 50, 1419-1432 (2007)

4. Baker, N., Cham, R., Cidboy, E.H., Cook, J., Redfern, M.S.: Kinematics of the fingers and hands during computer keyboard use. Clinical Biomechanics 22, 34-43 (2007)

5. Colle, H.A., Hiszem, K.J.: Standing at a kiosk: Effects of key size and spacing on touch screen numeric keypad performance and user preference. Ergonomics 47, 1406-1423 (2004)

6. Gong, J., Tarasewich, P.: Alphabetically Constrained Keypad Designs for Text Entry on Mobile Devices. In: CHI 2005, pp. 211-220. ACM Press, Portland (2005)

7. Sears, A., Zha, Y.: Data Entry for Mobile Devices Using Soft Keyboards: Understanding the Effects of Keyboard Size and User Tasks. International Journal of Human-Computer Interaction 16, 163-184 (2003)

8. MacKenzie, I.S., Zhang, S.X., Soukoreff, R.W.: Text entry using soft keyboards. Behaviour \& Information Technology 18, 235-244 (1999)

9. Zhai, S., Hunter, M., Smith, B.A.: The Metropolis keyboard-an exploration of quantitative techniques for virtual keyboard design. In: UIST 2000 Proceedings of the ACM Symposium on User Interface Software and Technology, pp. 119-128. ACM Press, San Diego (2000)

10. Goldstein, M., Book, R., Alsiö, G., Tessa, S.: Non-keyboard QWERTY touch typing: a portable input interface for the mobile user. In: CHI 1999, pp. 32-39. ACM Press, Pittsburgh (1999)

11. Sears, A., Jacko, J.A., Chu, J., Moro, F.: The role of visual search in the design of effective soft keyboards. Behaviour and Information Technology 20, 159-166 (2001)

12. Li, Y., Chen, L., Goonetilleke, R.S.: A heuristic-based approach to optimize keyboard design for single-finger keying applications. International Journal of Industrial Ergonomics 36, 695-704 (2006)

13. Kim, S., Sohn, M., Pak, J., Lee, W.: One-key keyboard: a very small QWERTY keyboard supporting text entry for wearable computing. In: 18th Australia conference on ComputerHuman Interaction: Design: Activities, Artefacts and Environments, pp. 305-308. ACM Press, Sydney (2006)

14. Wobbrock, J.O., Myers, B.A., Aung, H.H., LoPresti, E.F.: Text entry from power wheelchairs: edgewrite for joysticks and touchpads. In: 6th international ACM SIGACCESS conference on Computers and accessibility, pp. 110-117. ACM Press, Atlanta (2004)

15. Silfverberg, M., MacKenzie, I.S., Korhonen, P.: Predicting text entry speed on mobile phones. In: CHI 2000, pp. 9-16. ACM Press, Hague (2000)

16. Mizobuchi, S., Chignell, M., Newton, D.: Mobile text entry: relationship between walking speed and text input task difficulty. In: 7th international conference on Human computer interaction with mobile devices \& services, pp. 122-128. ACM Press, Salzburg (2005)

17. Jonsson, P., Johnson, P.W., Hagberg, M.: Accuracy and feasibility of using an electrogoniometer for measuring simple thumb movements. Ergonomics 50, 647-659 (2007) 